\title{
TOPICS IN PHYSICO-CHEMICAL HYDRODYNAMICS
}

\author{
PROGRESS REPORT \#2 \\ For the Period November 1, 1989 - October 31, 1990
}

\author{
Prepared for \\ THE U. S. DEPARTMENT OF ENERGY \\ DE-FGO2-88ER13822
}

\section{DISCLAIMER}

\begin{abstract}
This report was prepared as an account of work sponsored by an agency of the United States Government. Neither the United States Government nor any agency thereof, nor any of their employees, makes any warranty, express or implied, or assumes any legal liability or responsibility for the accuracy, completeness, or usefulness of any information, apparatus, product, or process disclosed, or represents that its use would not infringe privately owned rights. Reference herein to any specific commercial product, process, or service by trade name, trademark, manufacturer, or otherwise does not necessarily constitute or imply its endorsement, recommendation, or favoring by the United States Government or any agency thereof. The views and opinions of authors expressed herein do not necessarily state or reflect those of the United States Government or any agency thereof.
\end{abstract}

Prepared by:

Professor Gregory Sivashinsky

Principal Investigator

Levich Institute for Physico-Chemical Hydrodynamics

The City College of CUNY

Convent Avenue a 138 th street

New York, NY 10031 


\section{RESULTS ON PREMIXED COMBUSTION OBTAINED FROM PRIOR D.O.E. SUPPORT}

The variety of problems on premixed gas flames have been studied under DOE proposal No. DE-FG02-88ER13822. A brief account of the results obtained and a list of the publications supported by this grant follows.

\subsection{Theory of Turbulent Flame Speed.}

Turbulent flame propagation is modeled as a passive front moving through a multiple-scale flow-field, in which large-scale eddies convect small-scale eddies without causing their significant distortion. Assuming turbulence to be large-scaled the velocity of flame propagation relative to the underlying flow is regarded as a prescribed physico-chemical constant $U_{L}$. It is found that the temporal activity of the eddies composing the multiple-scale flow exert a damping influence on the flame front wrinkles. As a result, if the temporal activity of the eddies is low enough, the turbulent flame speed $U_{T}$ turns out to be proportional to the flow intensity $W_{R M S}$ provided $W_{R M S} \gg U_{l}$. Otherwise, $U_{T} \sim U_{L}^{1-a} W_{R M S}^{a}$, where $\alpha<1$.

For moderately high levels of turbulence $\left(W_{R M} s \lessgtr 6 U_{L}\right)$ the predictions of the theory correlate reasonably well with the experimental date available. More details may be found in Section (4.1) of this proposal.

These studies are reported in publications Nos. 1,2,3,4.

\subsection{Flame Extinction by Periodic Flow-Field.}

The present work is motivated by the observation that the premixed flame propagating through a turbulent flow is quenched when the intensity of turbulence is strong enough. To illustrate the mechanism of the phenomenon a simple flame-flow interaction scheme is proposed. In this model a time-independent spatially periodic tame-flow configuration is sustained by the underlying unidirectional periodic flow-field. The analysis, valid for $L e>1$ systems, show that the flame quenches, provided the amplitude (intensity) of the periodic flow exceeds a certain critical level. More details may be found in Section (4.2) of this proposal. 
This work is reported in publication No. 5 .

\subsection{Influence of Swirl on the Structure and Extinction of Premixed Flames.}

Premixed combustion processes of practical interest occur as a rule in turbulent flow.fields and involve complex flame-flow interactions. One of the basic interactions of this type is that imposed by the rotation of the gas flow crossing the flame front. The effects of rotation on the flame shape and it propagatability limits may be quite nontrivial.

In the present studies two classical systems are analyzed: the premixed flame stabilized(i) in the rotating Bunsen burner and (ii) in counterfowing streams with counter and corotating swirl.

It is shown that as the angular velocity of rotation increases, the classical Bunsen cone first slightly buckles at the tip. Buckling then becomes more prominent and eventually the flame enters the burner. At sufficiently rapid rotation flame stabilization by the burner rim becomes impossible and the flame flashes back assuming the shape of a downward moving paraboloid. The theoretical findings are sustained by experimental observations made on a rotating Bunsen burner.

This work is reported in publication No. 6.

Our interest in the counterflow systems was stimulated by the recent experimental studies of S. Sohrab and his co-workers in which counter-rotating swirl was imposed on back-to-back counter flow flames by spinning the exit ducts about their common axis in opposite directions. For suitably high rates of rotation the measured velocity profiles reveal recirculation regions with a radially inward flow in the neighborhood of the axis of rotation. In these experiments the lean flammability limits in methane-air and butane-air mixtures are found to decrease to a minimum and thereafter to increase as the rotation rate of the jets is increased and as the recirculation zone appears.

In our theoretical studies the counterflow system was analyzed both numerically and by activa. tion energy asymptotics for a one step Arrhenius reaction with small departures from unity Lewis numbers. Density changes due to heat release are fully taken into account. The results obtained capture all the major features of observations, including non-monotonicity of the dependency of the lean flammability limits on the reaction heat.

These works are reported in publications $N$ os. $i$ and 8 .

As a by-product of our preoccupation with rotating systems we also described i) the buoyancy sustained premixed flame propagating in the horizontal duct and ii) explained the phenomenon of nonplanar flame configurations in counterflow systems, observed in some experimental studies.

These works are reported in publications Nos. 9 and 10. 


\subsection{Propagation and Extinction of Nonsteady Spherical Flame Fronts.}

Outward and inward propagating spherical flame fronts are studied analytically by activation energy asymptotics and nl:merically using adaptive methods. Effects of Lewis number, curvature, heat loss and thermal expansion on propagation rates and extinction conditions are examined. Results are compared to recent experimental observations of expanding spherical flame fronts in mixtures near the flammability limits. It is found that many of the features of the experiment results are described by the proposed model.

With regard to the problem of the inward propagating spherical flame it is shown that at Lewis numbers exceeding unity the hurning rate and the flame speed gradually increase as the spherical flame approaches its center. At small Lewis numbers the picture is quite the opposite. The burning rate and the flame speed slow down and at some finite distance from the center the flame goes out. It is found that despite quenching, the fiame manages to consume all the fuel available. There is no residual reactant after extinction.

These works are reported in publications Nos. 11 and 12.

\subsection{Geometrically Invariant Formulation of the Intrinsic Dynamics of Premixed Flames.}

The range of validity of the existing weakly nonlinear equation for the intrinsic dynamics of the flame interface is limited only to mildly distorted planar flames.

The equation, therefore, is associated with a highly specialized system of coordinates. The present work was motivated by the desire to remove this restriction and to obtain a geometrically invariant formulation for the dynamics of tame evolution. The problem was successfully solved by neglecting the vorticity production at the flame interface* and assuming that the typicial radius of curvature of the flame is large compared with its thermo-diffusive width.

These studies are reported in publications. Nos. 13 and 14 .

\subsection{Nonlinear Dynamics of Oscillatory Regime of Premixed Combustion.}

Analytically obtaining nonlinear evolution equations for pulsating (and for spinninis) modes of flame propagation in Le $>1$ systems is a difficult problem of combustion theory.

The main technical obstacle here is the absence of any natural separation of scales in the starting equations; as a result, even though the flame evolution may often be considererl as "slow". a satisfactory formalism to handle these nonlinear systems has not yet been found.

\footnotetext{
* This is asymptotically correct when thermal expansion is weak.
} 
The present studies aim at achieving this goal. The new method proposed here consists . of embedding the original problem in a more general extended framework (via the introduction of an auxiliary parameter $\mu$ ) for which separation of scales naturally shows up. Upon use of the perturbation series about the bifurcation point in extended parameter space, an approximate evolution equation can be obtained. It is then extrapolated back to the original situation $(\mu=1)$. The obtained evolution equation possesses a number of intuitively desirable features (e.g. stable limit cycles) which hitherto were not feasible through conventional bifurcational analysis.

These studies are reported in publications Nos. 15 and 16.

\subsection{Pattern Formation in Premixed Flames.}

Spontaneous pattern formation is one of the most intriguing and challenging areas of classical phenomology. Despite considerable progress achieved in the understanding of one-dimensional systems, there are still many uncertainties with regard to the systems of higher dimensions. Open questions remain as to why certain periodic patterns are preferable to others and why periodic structures form at all. One such example may be found in the theory of flame propagation.

If molecular diffusivity of the deficient reactant exceeds thermal conductivity of the combustible mixture, the planar flarae looses its stability and spontaneously assumes a cellular structure. In suitably chosin dimeasions units the evolution equation for the perturbed downward propagating flame reads

$$
\Phi_{t}+\frac{1}{2}(\nabla \Phi)^{2}+2 \nabla^{2} \Phi+\nabla^{4} \Phi+G(\Phi-\langle\Phi\rangle)=0
$$

where $G$ is a scaled gravity acceleration.

The basic solution ( $\Phi=$ cont) is linearly stable or unstable depending on where $G$ is larger or smaller than unity.

The numerical solution of $\mathrm{Eq}$ (\#) shows that near the stability threshold $(G=1)$, instead of the time-dependent chaotically recombining cellular structure, known to occur in freely propagating flames $(G=0)$, the flame assumes a time-independent regular hexagonal pattern. Moreover, this hexagonal pattern survives even for the values of $G$ slightly above unity, indicating the subscribed nature of bifurcation at $G=1$. The numerical data are compared with the pertinent approximate analytical solution obtained by straightforward asymptotic expansion around $G=1$.

Under other geometric and hydrodynamic conditions the cellular flame may assume such shapes as a planar travelling wave or even a uniformly spinning regular polyhedral pyramid. Such a structure sometimes emerges on a Bunsen burner and is known to be the earliest observation of 
'cellular flame instability. Although the physical reasons for the polyhedral flames are quite clear, . the actual mathematical description of this striking phenomenon is still missing.

Recently, Buckmaster (SIAM J. Appl. Math. 1982) propoied an interesting bimodal bifurcational approach which made it possible to construct a set of travelling wave solutions for a one-dimensional variant of $\mathrm{Eq}(\#)$. An appropriate extension of Buckmaster's analysis permits description of spinning polyhedral structures sometimes observed in downward propagating flames in vertical tubes.

These studies of $E q(\#)$ are reported in publications Nos. 17 and 18.

It is well known that in the range of larger-scale perturbations the principal influence responsible for spontaneous flame instability is that of a purely hydrodynamic origin and conditioned by the thermal expansion of the burned gas. This effect is always present in large scale flames. In suitably chosen units the evolution equation describing dynamics of the perturbed planar flame in the region of hydrodynamic instability (Le $>1$ ) reads:

$$
\Phi_{t}+\frac{1}{2}(\nabla \Phi)^{2}=\Gamma^{2} \Phi+\frac{1}{4 \pi^{2}} \int|k| e^{i k\left(x-x^{\prime}\right)} \Phi(x, t) d k d x
$$

Previous numerical studies of this equation restricted to moderately wide domains, have shown that in the course of its evolution the flame assumes a single cusp configuration, irrespective of the initial data and size of the domain. This character of fame instability is in agreement with the known observations of small scale laboratory flames. However, experiments with unconfined large-scale flames and small-scale tames in high-pressure closed vessels indicate the emergence of a secondary (cellular) structure.

Stimulated by these observations more extensive numerical experiments with $\mathrm{Eq}(\#$ \#) bave been conducted. It is shown that while in sufficiently small-scale systems spontaneous instability appears in the guise of cusp-shaped flames - in larger-scale flames this configuration indeed acquires a fine cellular structure with the cells constantly subdividing and merging in a chaotic manner.

This work is reported in publication No. 19.

The considerable amount of progress achieved in the last fifteen years in the understanding of the intrinsic dynamics of premixed flames is reviewed in the solicited paper for the Proc. Roy. Soc. London. (Publication No. 20).

\subsection{Work in Progress.}

We are currently working on the following three projects. 
-". The first one deals with the evaluation of the effective transport coefficients in multiple-scale flow-fields. This study, apart from its own interest, is a necessary step towards the further devel. opment of the cascade models of turbulent combustion (cf. Section (4.1) of this proposal).

The second project is the formulation of the theory of slot burner polythedral flames. This work is conducted in collaboration with Professor C.K. Law of Princeton University.

The third project concerns a mathematical description of so-called "tulip" flames which are frequently observed in closed vessel combustion and which attracted much attention in recent years. This problem is studied in collaboration with Dr. G. Joulin of Universite de Poitiers, France.

We aim to complete these studies within the next six months so as to bring a successful conclusion to the present program.

\subsection{List of Journal Publications.}

1. "Cascade-Renormalization Theory of Turbulent Flame Speed," by G.I. Sivashinsky, Combustion Science and Technology, Vol. 68, pp. iT.96 (1988).

2. "Renormalization Concept of Turbulent Flame Speed" by G.I. Sivashinsky, Lecture .Votes in Physics, Numerical Simulation of Combustiori Phenomena (B. Larrouturou, ed.) SpringerVerlag (1990), to appear.

3. "Cascade Model of Turbulent Flame Propagation" by G.I. Sivashinsky, Lecture .Notes in Physics, Dissipative Structures in Transport Processes and Combustion (D.Meinköhn, ed.) Springer-Verlag (1990), to appear.

4. "Flame Propagation in Nonsteady Hydrodynamic fields" by Wm.I. Ashurst, G.I. Sivashinsky and V. Yakhot, Combustion Science and Technology, Vol. 62, Pp. 2i3-284 (1988).

5. "Flame Extinction by Periodic Flow.Field" by H. Berestycki and G.I. Sivashinsky, SIA.M J. on Applied Mathematics (1990), submitted.

6. "Effects of Rutation on Bunsen Flame" by W.J. Sheu, S.H. Sohrab and G.I. Sivashinsky, Combustion and Flame (1990), to appear.

7. "Influences of Swirl on the Structure and Extinction of Strained Premixed Flames" by P. Libby. G.I. Sivashinsky and F.A. Williams, Physics of Fluids (1990), submitted.

8. "Aerodynamics of Viscous Flow in Counter-Rotating Finite Jets" by W.J. Sheu, S.H. Sohrab and G.I. Sivashinsky, Combustion Science and Technology (1990) sujumitted.

9. "Flame Propagation in th: Horizontal Channel" by Z. Rakib and G.I. Sivashinsky, Combustion Science and Technology, Vol. 59, pp. 247.260 (1988). 
10. "Nonplanar Flane Configurations in Stagnation Point Flow", by W.J. Sheu and G.I. Sivashinsky, Combustion and Flame (1990), to appear.

11. "A Theoretical Study of Propagation and Extinction of Nonsteady Spherical Flame Fronts", by P. Ronney and G.I. Sivashinsky, SI.A.M J. on Applied Mathematics, Vol. 49, pp. 1029.50 (1989).

12. "Numerical Study of Curvature Effects on Flame Quenching", by G. Fenandez, B. Larrouturou and G.I. Sivashinsky, Lecture Notes in Physics, Vumerical Simulation of Combustion Phenom. ena (B. Larrouturou, ed), Springer-Verlag (1990), to appear.

13. "On the Equation of a Curved Flame Front", by M. Frankel and G.I. Sivashinsky, Physica D, Nonlinear Phenomena, Vol. 30, pp. $28-42$ (1988).

14. "An Equation of Surface Dynamics Modelling the Thermal-Expansion Driven Flame Fronts", by M. Frankel, Physics of Fluids (1990), submitted.

15. "On the Equation of Pulsating Flame Fronts in Solid Fuel Combustion", by M. Frankel, Physica D, Nonlinear Phenomena (1990), submitted.

16. "Qualitative Approximation of Oscillatory Flames in Premixed Gas Combustion by a Local Equation of Front Dynamics", by M. Frankel, SIAM J. on Applied Mathematics (1990), submitted.

17. "On Hexagonal Structure of Cellular Flames", by L. Shtilman and G.I. Sivashinsky, Canadian J. of Physics (1990), to appear.

18. "On Spinning Propagation of Cellular Flames", by S. Margolis and G.I. Sivashinsky, Combustion Science and Technology (1990), to appear.

19. "The Cellular Nature of Hydrodynamic Flame Instability", by S. Gutman and G.I. Sivashinsky, Physics D, Nonlinear Phenomena (1990), to appear.

20. "Some Developments in the Nonlinear Dynamics of Premixed Flames", by G.I. Sivashinsky, Proceedings of the Royal Society (London) (1990), to appear. 

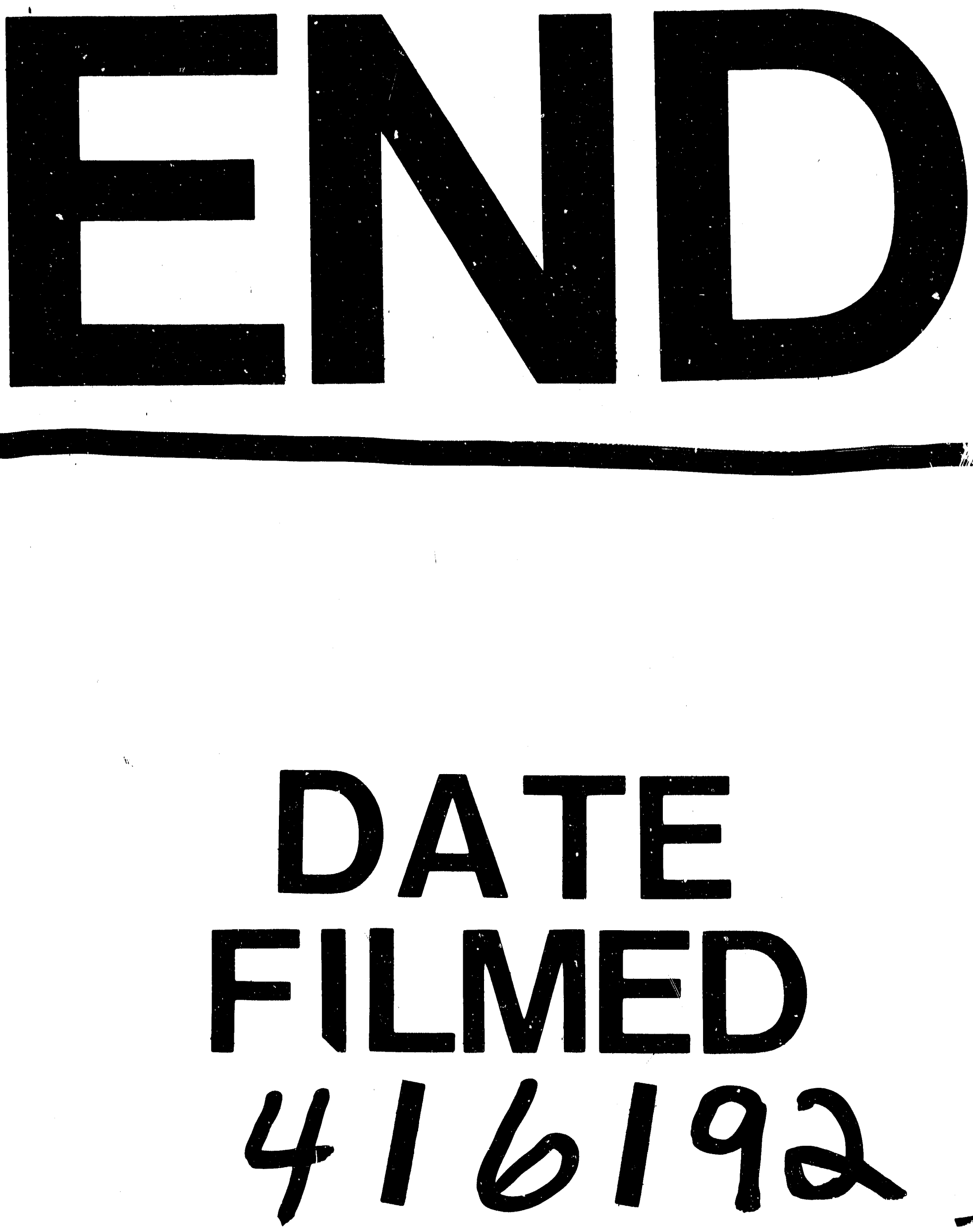

$I$ 
